\title{
FINITE GROUPS WITH SOME $\mathfrak{Z}$-PERMUTABLE SUBGROUPS*
}

\author{
YANGMING LI \\ Department of Mathematics, Guangdong Institute of Education, Guangzhou 510310, China \\ e-mail: liyangming@gdei.edu.cn \\ LIFANG WANG \\ School of Mathematics and Computer, Shanxi Normal University, Linfen 041004, China \\ e-mail: lfwang_2003@yahoo.com.cn \\ and YANMING WANG \\ Lingnan College and Department of Mathematics, Zhongshan University, Guangzhou 510275, China \\ e-mail:stswym@mail.sysu.edu.cn
}

(Received 13 April 2007; accepted 28 July 2009)

\begin{abstract}
Let $\mathfrak{Z}$ be a complete set of Sylow subgroups of a finite group $G$; that is to say for each prime $p$ dividing the order of $G, \mathfrak{Z}$ contains one and only one Sylow $p$-subgroup of $G$. A subgroup $H$ of $G$ is said to be $\mathfrak{Z}$-permutable in $G$ if $H$ permutes with every member of $\mathfrak{Z}$. In this paper we characterise the structure of finite groups $G$ with the assumption that (1) all the subgroups of $G_{p} \in \mathfrak{Z}$ are $\mathfrak{Z}$-permutable in $G$, for all prime $p \in \pi(G)$, or (2) all the subgroups of $G_{p} \cap F^{*}(G)$ are 3 -permutable in $G$, for all $G_{p} \in \mathfrak{Z}$ and $p \in \pi(G)$, where $F^{*}(G)$ is the generalised Fitting subgroup of $G$.
\end{abstract}

2000 Mathematics Subject Classification. 20D10, 20 D20.

1. Introduction and statements of results. All groups considered in this paper are finite. We use conventional notions and notation, as in Huppert [5]. Throughout this paper, $G$ stands for a finite group and $\pi(G)$ represents the set of distinct primes dividing $|G|$.

A subgroup of $G$ is called quasi-normal in $G$ if it permutes with every subgroup of $G$. We say, following Kegel [8], that a subgroup of $G$ is $S$-quasi-normal in $G$ if it permutes with every Sylow subgroup of $G$. Recently, Asaad and Heliel [1] introduced a new embedding property, namely the $\mathfrak{Z}$-permutability of subgroups of a group; $\mathfrak{Z}$ is called a complete set of Sylow subgroups of $G$ if for each prime $p \in \pi(G), \mathfrak{Z}$ contains exactly one Sylow $p$-subgroup of $G$, say $G_{p}$. A subgroup of $G$ is said to be $\mathfrak{Z}$-permutable in $G$ if it permutes with every member of $\mathfrak{Z}$. Obviously, every $S$-quasi-normal subgroup is $\mathfrak{Z}$-permutable. In contrast to the fact that every $S$-quasi-normal subgroup is subnormal (see [8]), it does not hold in general that every $\mathfrak{Z}$-permutable subgroup of $G$ is subnormal in $G$. It suffices to consider the alternating group of degree 4.

Many authors have investigated the structure of a group $G$ under the assumption that some subgroups of $G$ are well situated in $G$. Srinivasan [14] proved that a group $G$ is supersolvable if every maximal subgroup of any Sylow subgroup of $G$ is normal. Later on, Wall [15] gave a complete classification of finite groups under the assumption

* Project supported in part by NSFC(10871210), NSF of Guangdong (006023728), NSF for youth of Shanxi Province (2007021004) and Tian YuanFund of Mathematics of China (10726002). 
of Srinivasan. In [10], the authors obtained the following results ([10], Theorems 3.1 and 3.4): Let $\mathfrak{Z}$ be a complete set of Sylow subgroups of a group $G$ and $p$ the smallest prime dividing $|G|$. Then $G$ is $p$-nilpotent if one of the following hold: (1) the maximal subgroups of $G_{p} \in \mathfrak{Z}$ are $\mathfrak{Z}$-permutable subgroups of $G$; (2) $G$ is $A_{4}$-free and the 2maximal subgroups of $G_{p}$ are $\mathfrak{Z}$-permutable subgroups of $G$. In [11], the authors obtained the following ([11], Theorems 3.1 and 3.4): Let $\mathfrak{Z}$ be a complete set of Sylow subgroups of a group $G$ and $p$ the smallest prime dividing $|G|$. Then $G$ is $p$-nilpotent if one of following holds: (1) every cyclic subgroup of prime order or order 4 (when $p=2$ ) of $G_{p} \in \mathfrak{Z}$ is $\mathfrak{Z}$-permutable in $G$; (2) $G$ is $A_{4}$-free and every subgroup of prime square order of $G_{p} \in \mathfrak{Z}$ is $\mathfrak{Z}$-permutable in $G$. We know that if every subgroup of $G$ is normal in $G$; then $G$ is the Dedikind group [13]; every subgroup of $G$ is quasi-normal in $G$; then $G$ is the quasi-Hamilton group [2]. It is easy to see that that $G$ is nilpotent if and only if every subgroup of $G$ of prime power order is $\mathfrak{Z}$-permutable in $G$, where $\mathfrak{Z}$ is a complete set of Sylow subgroups of $G$. In view of the above results, it is interesting to give the structure of $G$ under the assumption that for any $G_{p} \in \mathfrak{Z}$, every subgroup of $G_{p}$ is $\mathfrak{Z}$-permutable in $G$. We get the following.

THEOREM 1.1. Let $G$ be a finite group and $\mathfrak{Z}$ a complete set of Sylow subgroups of $G$. Then every subgroup of $G_{p} \in \mathfrak{Z}$, for any prime $p \in \pi(G)$, is $\mathfrak{Z}$-permutable in $G$ if and only if there exists a normal subgroup $L$ of $G$ satisfying the following:

(1) $L$ is an abelian Hall subgroup of $G$ and $G / L$ is nilpotent;

(2) the elements of $G$ induce power automorphisms in $L$;

(3) for any two distinct primes $p, q \notin \pi(L),\left[G_{p}, G_{q}\right]=1$, where $G_{p}, G_{q} \in \mathfrak{Z}$.

It is interesting to limit the hypotheses to a smaller subgroup of $G$. By [4] and [9], we know the following: Let $G$ be a finite group and $\mathfrak{Z}$ a complete set of Sylow subgroups of $G$ and $F^{*}(G)$ is the generalised Fitting subgroup of $G$. Then $G$ is supersolvable under one of following assumptions: (1) the maximal subgroups of $G_{p} \cap F^{*}(G)$ are $\mathfrak{Z}$-permutable subgroups of $G$, for all $G_{p} \in \mathfrak{Z}$; (2) the cyclic subgroups of $G_{p} \cap F^{*}(G)$ of prime order or order are $\mathfrak{Z}$-permutable subgroups of $G$, for all $G_{p} \in \mathfrak{Z}$. Hence, it is interesting to investigate the structure of $G$ under the assumption that all the subgroups of $G_{p} \cap F^{*}(G)$ are $\mathfrak{Z}$-permutable subgroups of $G$, for all $G_{p} \in \mathfrak{Z}$. Here we get the following.

THEOREM 1.2. Let $G$ be a finite group and $\mathfrak{Z}$ a complete set of Sylow subgroups of $G$, and $F^{*}(G)$ is the generalised Fitting subgroup of $G$. Then every subgroup of $G_{p} \cap F^{*}(G)$, for any $G_{p} \in \mathfrak{Z}$ and any $p \in \pi(G)$, is $\mathfrak{Z}$-permutable in $G$ if and only if there exists a normal subgroup $L$ of $G$ satisfying the following:

(1) $L$ is abelian and $G / L$ is nilpotent;

(2) $L$ is a Hall subgroup of $F^{*}(G)$;

(3) $p^{\prime}$-elements of $G$ induce power automorphisms in $L_{p}$, the Sylow p-subgroup of $L$.

COROLLARY 1.3. Let $G$ be a finite group, and $F^{*}(G)$ is the generalised Fitting subgroup of $G$. Then every subgroup of $F^{*}(G)$ is $S$-quasi-normal in $G$ if and only if there exists a normal subgroup $L$ of $G$ satisfying the following:

(1) $L$ is abelian and $G / L$ is nilpotent;

(2) $L$ is a Hall subgroup of $F^{*}(G)$;

(3) $p^{\prime}$-elements of $G$ induce power automorphisms in $L_{p}$, the Sylow p-subgroup of $L$. 
Let $\mathfrak{Z}$ be a complete set of Sylow subgroups of a group $G$. If $N \triangleleft G$, we denote

$$
\begin{aligned}
\mathfrak{Z} N & =\left\{G_{p} N: G_{p} \in \mathfrak{Z}\right\}, \\
\mathfrak{Z} N / N & =\left\{G_{p} N / N: G_{p} \in \mathfrak{Z}\right\}, \\
\mathfrak{Z} \cap N & =\left\{G_{p} \cap N: G_{p} \in \mathfrak{Z}\right\} .
\end{aligned}
$$

The generalised Fitting subgroup $F^{*}(G)$ of $G$ is the unique maximal normal quasinilpotent subgroup of $G$. Its important properties can be found in [7], Chapter X, Section 13.

Now, $G^{\mathcal{N}}$ denotes the nilpotent residual of $G$, which some authors prefer to write as $K_{\infty}(G)$; it is the last term in the lower central series of $G$.

2. Preliminaries. The following lemmas will be used in the proofs of our results.

Lemma 2.1. ([1], Lemma 2.1) Let $\mathfrak{Z}$ be a complete set of Sylow subgroups of G, $U$ a $\mathfrak{Z}$-permutable subgroup of $G$ and $N$ a normal subgroup of $G$. Then

(1) $\mathfrak{Z} \cap N$ and $\mathfrak{Z} N / N$ are complete sets of Sylow subgroups of $N$ and $G / N$, respectively;

(2) $U N / N$ is a $\mathfrak{Z} N / N$-permutable subgroup of $G / N$;

(3) $U$ is a $\mathfrak{Z} \cap N$-permutable subgroup of $N$ if $U \leq N$.

LEMMA 2.2. Let $G$ be a finite group and $\mathfrak{Z}$ a complete set of Sylow subgroups of $G$. Suppose $N$ is a normal p-subgroup of $G$; then every subgroup of $N$ is $\mathfrak{Z}$-permutable in $G$ if and only if every subgroup of $N$ is $S$-quasi-normal in $G$.

Proof. We only need to prove the necessity. Suppose any subgroup of $N$ is $\mathfrak{Z}$ permutable in $G$. Let $L$ be an arbitrary subgroup of $N$. Then $L G_{p_{i}}$ is a subgroup of $G$ for every $G_{p_{i}} \in \mathfrak{Z}$. Since $N \triangleleft G$, it follows that $L \leq N^{x}$ for all $x \in G$. Hence $L^{x^{-1}} \leq N$, and therefore $L^{x^{-1}} G_{p_{i}} \leq G$. But $L G_{p_{i}}^{x}=\left(L^{x^{-1}} G_{p_{i}}\right)^{x}$ is a subgroup of $G$, then $L$ is $S$ quasi-normal in $G$.

Lemma 2.3. ([7]; Chapter $X$, Section 13) Let $G$ be a group and $M$ a subgroup of $G$.

(1) If $M$ is normal in $G$, then $F^{*}(M) \leq F^{*}(G)$.

(2) If $F^{*}(G)$ is solvable, then $F^{*}(G)=F(G)$.

LEMMA 2.4. Let $G$ be a finite group and $\mathfrak{Z}$ a complete set of Sylow subgroups of $G$. Suppose every subgroup of $F^{*}(G) \cap G_{p}$ is $\mathfrak{Z}$-permutable in $G$, for any $G_{p} \in \mathfrak{Z}$; then $G$ is supersolvable.

Proof. This is a corollary of results in [4] or [9].

LeMma 2.5. Suppose $G$ is a group and $P$ a normal $p$-subgroup of $G$. Then $P \leq Z_{\infty}(G)$ if and only if $C_{G}(P) \geq O^{p}(G)$.

Proof. If $C_{G}(P) \geq O^{p}(G)$, then $G / C_{G}(P)$ is a $p$-group; so $P \leq Z_{\infty}(G)$ by [16], p. 220, Theorem 6.3. The converse is [12], Lemma 2.8 .

Lemma 2.6. Suppose $P$ is a normal p-subgroup of $G$. If every subgroup of $P$ is $S$-quasi-normal in $G$, then every $p^{\prime}$-element of $G$ induces a power automorphism in $P$. 
Proof. Take any $a \in P$. Let $x$ be a $p^{\prime}$-element of $G$. Then $x \in G_{p^{\prime}}$ for some $p^{\prime}$-Hall subgroup of $G$. Then $\langle a\rangle G_{p^{\prime}}$ is a group by hypotheses. Hence

$$
a^{\langle x\rangle}=a^{\langle x\rangle} \cap\langle a\rangle G_{p^{\prime}}=\langle a\rangle\left(a^{\langle x\rangle} \cap G_{p^{\prime}}\right) \leq\langle a\rangle\left(P \cap G_{p^{\prime}}\right)=\langle a\rangle,
$$

i.e. $a^{\langle x\rangle}=\langle a\rangle$. Therefore $x$ induces a power automorphism in $\langle a\rangle$.

In the next lemma we collect some properties of power automorphism.

Lemma 2.7. Suppose $N$ is a non-trivial normal p-subgroup of $G$. Then

(1) the $p^{\prime}$-power automorphism of $N$ is trivial if $N$ is non-abelian;

(2) there exists a positive integer $n$ such that $a^{\alpha}=a^{n}$, for all $a \in N$, if $N$ is abelian and $\alpha$ is a power automorphism of $N$;

(3) the power automorphisms of $N$ are in the centre of $\operatorname{Aut}(N)$ if $N$ is abelian;

(4) $G / C_{G}(N)$ is nilpotent if all $p^{\prime}$-elements of $G$ induce power automorphisms in $N$ by conjugate.

Proof. (1) It is [6], Hilfsatz 5.

(2) See [13], Chapter 13, Theorem 4.3.

(3) It is a direct corollary of (2).

(4) If $N$ is non-abelian, then $G / C_{G}(N)$ is a $p$-group by (1); hence $G / C_{G}(N)$ is nilpotent. If $N$ is abelian, then the power automorphisms are in the centre of $\operatorname{Aut}(N)$ by (3). It is easy to see that $G / C_{G}(N)$ is nilpotent.

Lemma 2.8. Suppose $L=K_{\infty}(G)$ is the nilpotent residual of $G$. If $L$ is nilpotent, then $L_{p}=\left[L_{p}, G\right]$, for any $p \in \pi(G)$.

Proof. By definition, $L=[L, G]=\left[L_{p} \times L_{p^{\prime}}, G\right]=\left[L_{p}, G\right] \times\left[L_{p^{\prime}}, G\right]=L_{p} \times L_{p^{\prime}}$, for any $p \in \pi(G)$. Hence $L_{p}=\left[L_{p}, G\right]$.

\section{Proofs.}

Proof of Theorem 1.1. We prove the necessity of this theorem in several steps.

(i) $G$ is supersolvable. Hence $F^{*}(G)=F(G)$.

By Lemmata 2.4 and 2.3.

(ii) If $N$ is a normal $p$-subgroup of $G$, then $p^{\prime}$-elements of $G$ induce power automorphisms in $N$.

If $N$ is a normal $p$-subgroup of $G$, then $N \leq G_{p} \in \mathfrak{Z}$. Thus every subgroup of $N$ is $\mathfrak{Z}$-permutable in $G$ by hypotheses; then is $S$-quasi-normal in $G$ by Lemma 2.2. Now applying Lemma 2.6, we get step (ii).

(iii) Pick $L=G^{\mathcal{N}}$; then $G / L$ is nilpotent. Furthermore, $L$ is abelian.

By (ii) and Lemma 2.7(4), for any $p \in \pi(G)$, we know that $G / C_{G}\left(O_{p}(G)\right)$ is nilpotent. So $\left(G / C_{G}\left(O_{p}(G)\right)\right)^{\mathcal{N}}=1$, and it follows that $G^{\mathcal{N}} \leq C_{G}\left(O_{p}(G)\right)$. Therefore $G^{\mathcal{N}} \leq \bigcap_{p \in \pi(G)} C_{G}\left(O_{p}(G)\right)=C_{G}(F(G)) \leq F(G)$. Then $L \leq Z(F(G))$, Hence $L$ is abelian.

(iv) $L$ is a Hall subgroup of $G$.

Let $p$ be the largest prime dividing $|G|$, and $P$ is a Sylow $p$-subgroup of $G$. Since $G$ is supersolvable by step (i), we know that $P \unlhd G$. Then $P=G_{p} \in \mathfrak{Z}$. Now, we consider the quotient group $G / P$. By Lemma 2.1, all subgroups of every member in $\mathfrak{Z} P / P$ are $\mathfrak{Z} P / P$-permutable in $G / P$. By induction, $(G / P)^{\mathcal{N}}=G^{\mathcal{N}} P / P=L P / P$ is a Hall subgroup of $G / P$. 
Suppose that every $p^{\prime}$-element of $G$ centralises $P$. Let $L_{p} \in S y l_{p}(L)$. If $L_{p} \neq 1$, then, by Lemma 2.8, $L_{p}=\left[L_{p}, G\right]=\left[L_{p}, P\right]<L_{p}$ as $L_{p} \leq P$, a contradiction. Hence $L_{p}=1$ and $L$ is a $p^{\prime}$-group. Therefore, $L \cong L P / P$ is a normal Hall subgroup of $G$. Now suppose that there exists a $p^{\prime}$-element $x$ which induces a non-trivial power automorphism on $P$. Hence $P=[P, G] \leq L$. Therefore $L$ is a Hall subgroup of $G$.

(v) The elements of $G$ induce power automorphisms in $L$.

It is easy to see from (ii)-(iv).

(vi) For any two distinct primes $p, q \notin \pi(L),\left[G_{p}, G_{q}\right]=1$, where $G_{p}, G_{q} \in \mathfrak{Z}$.

By the hypotheses, $G_{p} G_{q}$ is a group. Since $G_{p} G_{q} \cong G_{p} G_{q} L / L \leq G / L, G_{p} G_{q}$ is nilpotent by (iii). Hence $\left[G_{p}, G_{q}\right]=1$.

Conversely, it suffices to prove that $\langle x\rangle\langle y\rangle=\langle y\rangle\langle x\rangle$, for any $p$-element $x \in G_{p}$ and $q$-element $y \in G_{q}$, where $G_{p}, G_{q} \in \mathfrak{Z}$.

If $p, q \in \pi(L)$, since $L$ is a normal abelian Hall subgroup of $G$, we have that $x, y \in L$ and $\langle x\rangle\langle y\rangle=\langle y\rangle\langle x\rangle$.

If $p, q \notin \pi(L)$, by (3), $[x, y]=1$. Hence $\langle x\rangle\langle y\rangle=\langle y\rangle\langle x\rangle$.

Suppose $p \in \pi(L)$ or $q \in \pi(L)$. Without lose generality, let $p \in \pi(L)$. Then $x \in L$ and $\langle x\rangle \unlhd G$ by (2). Hence $\langle x\rangle\langle y\rangle=\langle y\rangle\langle x\rangle$. Finishing the proof.

Proof of Theorem 1.2. We first prove the necessity of Theorem 1.2. With the same arguments as in the proof of Theorem 1.1, we get steps (i)-(iii).

(i) $G$ is supersolvable. Hence $F^{*}(G)=F(G)$.

(ii) If $N$ is a normal $p$-subgroup of $G$, then $p^{\prime}$-elements of $G$ induce power automorphisms in $N$.

(iii) Pick $L=G^{\mathcal{N}}$; then $G / L$ is nilpotent. Furthermore, $L$ is abelian.

(iv) Denote $F=F(G)$. Then $F=C_{G}(L)$.

By the proof of (iii), we only need to prove $C_{G}(L) \leq F$. We know that $L \leq$ $Z\left(C_{G}(L)\right)$, and hence $C_{G}(L) / Z\left(C_{G}(L)\right) \leq G / Z\left(C_{G}(L)\right)$ is nilpotent. Hence $C_{G}(L)$ is nilpotent. Therefore $C_{G}(L) \leq F(G)$.

(iv) $F=Z_{\infty}(G) \times L$.

By [5], Chapter VI, Satz 7.15, $G$ splits over $L$, i.e. $G=X \triangleright<L$, for some subgroup $X$ of $G$. So $F=F \cap(X L)=L\left(C_{G}(L) \cap X\right)=C_{X}(L) L=C_{X}(L) \times L$.

Now we prove that $C_{X}(L)=Z_{\infty}(G)$.

Notice that $\left[C_{X}(L), G\right]=\left[C_{X}(L), X\right] \leq C_{X}(L)$. Since $X \cong G / L$ is nilpotent, there exists an integer $n$ such that $K_{n}(X)=K_{\infty}(X)=1$. Therefore $\left[C_{X}(L), G, \ldots, G\right]=\left[C_{X}(L), X, \ldots, X\right] \leq K_{\infty}(X)=1$. Therefore $C_{X}(L) \leq Z_{n-2}(G) \leq$ $Z_{\infty}(G)$. Thus $Z_{\infty}(G)=Z_{\infty}(G) \cap F=C_{X}(L)\left(Z_{\infty}(G) \cap L\right)$,

Next we want to prove that $Z_{\infty}(G) \cap L=1$. If $Z_{\infty}(G) \cap L \neq 1$, then there exists a prime $p \in \pi(G)$ such that $Z_{\infty}(G) \cap L_{p} \neq 1$. If every $p^{\prime}$-element of $G$ centralises $L_{p}$, then, by Lemma 2.8, $L_{p}=\left[L_{p}, G\right]=\left[L_{p}, G_{p}\right]<L_{p}$, a contradiction. Hence there exists a $p^{\prime}-$ element $x$ which induces a non-trivial power automorphism on $L_{p}$, and so $\left[L_{p}, x\right] \neq 1$. On the other hand, we know that $\left[Z_{\infty}(G) \cap L_{p}, x\right]=1$ by Lemma 2.5 . Hence $\left[L_{p}, x\right]=1$ by (iii) and Lemma 2.7(2), a contradiction. Hence $Z_{\infty}(G) \cap L=1$. So $Z_{\infty}(G)=C_{X}(L)$. Thus $F=Z_{\infty}(G) \times L$.

(v) $L$ is a Hall subgroup of $F(G)$.

If $L$ is not a Hall subgroup of $F(G)$, then there is a prime $p \in \pi(L) \cap \pi(F(G) / L)$. Denote $C=C_{X}(L)$.

By (iv), $F=C_{G}(L)=Z_{\infty}(G) \times L=C_{X}(L) \times L=C \times L$. Since $p \in \pi(C)$, we have $C_{p} \neq 1$; then $C_{p} \cap Z(C) \neq 1$. Therefore $p \in \pi(Z(C))$. For any $p^{\prime}$-element $x \in X, x$ 
induces a power automorphism on $F_{p}=C_{p} \times L_{p}$. By Lemma 2.5, $\left[C_{p}, x\right]=1$. Hence $\left[Z\left(C_{p}\right), x\right]=1$. By Lemma 2.7(2), $\left[Z\left(C_{p}\right) \times L_{p}, x\right]=1$, and in particular, $\left[L_{p}, x\right]=1$. Therefore $\left[L_{p}, X_{p^{\prime}}\right]=1$. Then $L_{p}=\left[L_{p}, G\right]=\left[L_{p}, X\right]=\left[L_{p}, X_{p}\right]<L_{p}$, a contradiction. Therefore $L$ is a Hall subgroup of $F(G)$.

Conversely, it is easy to see that $G$ is solvable; hence $F^{*}(G)=F(G) \neq 1$. It suffices to prove that every cyclic $p$-subgroup $<g>$ of $F(G)$ is $S$-quasi-normal in $G$, for any prime $p \in \pi(G)$.

Suppose $g \in O_{p}(G)$. If $P \in S y l_{p}(G)$, then $g \in O_{p}(G) \leq P$. Thus $<g>P=P<$ $g>=P$. Pick an arbitrary $Q \in S y l_{q}(G)$, where $q \neq p$. If $p \in \pi(L)$, then $g \in L_{p}$ by (iii). Then $Q$ normalises $<g>$ by (ii). Therefore $Q<g>=<g>Q$. Hence suppose that $p \notin \pi(L)$. Then $[<g>L / L, Q L / L]=1$ as $G / L$ is nilpotent by (i). So $[<g>, Q] \leq L$. It follows that $[Q, g] \leq L \cap g^{G} \leq L \cap O_{p}(G)=1$. Hence $Q<g>=<g>Q$. Completing the proof.

Proof of Corollary 1.3. If every subgroup of $F^{*}(G)$ is $S$-quasi-normal in $G$, then $G$ is supersolvable by the results in [4] or [9]. In particular, $F^{*}(G)=F(G)$. For any prime $p \in \pi(G)$ and any Sylow $p$-subgroup $G_{p}$ of $G$, every subgroup of $G_{p} \cap F^{*}(G)=$ $G_{p} \cap F(G)=O_{p}(G)$ is $S$-quasi-normal in $G$ by the hypotheses. By Theorem 1.2, it is easy to see the necessity of Corollary 1.3 holds. Conversely, by the proof of Theorem 1.2 , we know that every cyclic $p$-subgroup of $F(G)=F^{*}(G)$ is $S$-quasi-normal in $G$, for any prime $p \in \pi(G)$. It is easy to see that every subgroup of $F^{*}(G)$ is $S$-quasi-normal in $G$. Completing the proof.

\section{REFERENCES}

1. M. Asaad and A. A. Heliel, On permutable subgroups of finite groups, Arch. Math. 80 (2003), 113-118.

2. Pic Gheorghe, On the structure of Quasi-Hamilton group, Acad. Repub. Pop. Române. Sti. 1(1949), 973-979.

3. D. Gorenstein, Finite simple groups (Plenum, New York, 1982).

4. A. A. Heliel, Xianhua Li and Yangming Li, On $\mathfrak{Z}$-permutability of minimal subgroups, Arch Math. 83(2004), 9-16.

5. B. Huppert, Endliche Gruppen I (Springer, Berlin, 1968).

6. B. Huppert, Zur Sylowstruktur Auflösbarer Gruppen, Arch. Math. 12(1961), 161-169.

7. B. Huppert and N. Blackburn, Finite groups III (Springer, Berlin, 1982).

8. O. Kegel, Sylow-Gruppen und subnormalteiler endlicher Gruppen, Math. Z. 78(1962), 205-221.

9. Y. Li and A. A. Heliel, On permutable subgroups of finite groups, Comm. Algebra 33(9) (2005), 3353-3358.

10. Y. Li and X. Li, $\mathfrak{Z}$-permutable subgroups and $p$-nilpotency of finite groups, J. Pure Appl. Algebra 202 (2005), 72-81.

11. X. Li, Y. Li and L. Wang, $\mathfrak{Z}$-permutable subgroups and p-nilpotency of finite groups II, Isr. J. Math. 164 (2008), 75-85.

12. Y. Li and Yanming Wang, On $\pi$-quasinormally embedded subgroups of finite group, $J$. Algebra 281 (2004), 109-123.

13. D. J. S. Robinson, A course on the theory of groups (Springer, Berlin, 1980).

14. S. Srinivasan, Two sufficient conditions for supersolvability of finite groups, Isr. J. Math. 35 (1980), 210-214.

15. G. Wall, Groups with maximal subgroups of Sylow subgroups normal, Isr. J. Math. $\mathbf{4 3}$ (1982), 166-168.

16. M. Weinstein (Editor), Between nilpotent and solvable (Polygonal, Passaic, Washington, NJ, USA, 1982). 